\title{
Conserve Energy through Multiple-PaCkets Transmission in Heterogeneous Mobile AD Hoc NeTWORKS
}

\author{
Wei Liu, Yanchao Zhang, and Yuguang Fang \\ Department of Electrical and Computer Engineering \\ University of Florida \\ Gainesville, Florida 32611 \\ Email:\{liuw@,yczhang@,fang@ece.\}ufl.edu
}

\begin{abstract}
Energy conservation is a crucial issue in Mobile Ad Hoc Networks (MANETs). In our previous work, we have proposed a Device-Energy-Load Aware Relaying framework (DELAR) [1] that can effectively utilize some powerful nodes ( $P$-nodes) to conserve energy for other common nodes (B-nodes) and in turn prolong the network lifetime. DELAR is a joint design of power control, routing, and scheduling. Moreover, in this framework, an Asymmetric MAC (A-MAC) is adopted to support reliable communications on unidirectional links caused by asymmetric transmission power between the involved transmitter and receiver. Grounded on this DELAR framework which emphasizes the interaction between the MAC layer and the network layer, in this paper we further take into account the physical layer and propose a multiple-packets transmission scheme which is jointly operated with hierarchical modulation to further improve the energy efficiency and shorten the packet delay. The basic idea is to enable such P-nodes to transmit multiple packets towards different receivers in one transmission, and this transmission is implemented with hierarchical modulation to ensure sufficient SNR at all the receivers to demodulate their corresponding packets. Simulation results validate the effectiveness and efficiency of this multiple-packets transmission scheme: It can further improve the energy efficiency and greatly shorten the packet delay.
\end{abstract}

\section{INTRODUCTION}

Mobile Ad Hoc Networks (MANETs) is one of the indispensable components to support ubiquitous communications. To make the wide-scale deployment of MANETs possible, extensive research efforts are currently underway to enhance the operation and management of such networks. One of the major challenges comes from nodes' finite energy supply: A MANET usually consists of battery-powered mobile devices (nodes) which would become useless once their limited energy reserve is depleted. Some devastating consequences of such node diminution include the degrada- tion of network performance, unfavorable network partition, and so on. As a result, the energy conservation of mobile devices becomes a crucial issue for the realistic deployment of MANETs.

The heterogeneity of mobile devices seems to be inherent and has been commonly observed in MANETs [2]. Therefore, different from many previous works assuming nodes are homogeneous, we consider more realistic networks in which nodes are heterogeneous in one or more aspect. In particular, we are interested in such heterogeneous ad hoc networks in terms of energy supply, where most nodes, denoted as B-nodes, are powered by batteries with small capacity, while some other nodes, denoted as P-nodes, are powered by batteries with much larger capacity compared to those B-nodes, or by recoverable energy sources, e.g., power scavenging units such as solar cells, or by dynamos when they are installed in mobile vehicles. Our intention is to develop more energy conscious protocols by taking advantage of such heterogeneity of mobile devices to conserve energy and in turn prolong the network lifetime, i.e., being generous in using the P-nodes while conservative in using the B-nodes.

In our previous work [1], we proposed a cross-layer designed Device-Energy-Load Aware Relaying framework (DELAR) [1] that effectively utilizes such P-nodes to conserve energy for other B-nodes and in turn prolong the network lifetime. DELAR is a joint design of scheduling, power control, and routing. By dividing time into superframes each of which consists of multiple periods, the transmission activities of P-nodes and B-nodes are coordinated by a hybrid of reservation-based MAC and contention-based MAC protocols. In order to make use of Pnodes' special feature of energy supply, P-nodes are enabled to use different transmission power in different periods. In addition, the information about residue energy, local load status, and device property such as energy supply, is nicely incorporated into routing protocols to find energy-efficient paths. Moreover, in this framework a novel Asymmetric MAC (A-MAC) is adopted to support reliable communications on unidirectional links caused by asymmetric 
transmission power between the involved transmitter and receiver. DELAR can greatly improve the performance of energy efficiency, however, at the cost of prolonged packet delay due to the employed transmission scheduling. Grounded on this DELAR framework which emphasizes the interaction between the MAC and network layers, in this paper we further take into account the physical layer and propose a multiple-packets transmission scheme which is jointly operated with hierarchical modulation to further improve the energy efficiency. More important, by incorporating into DELAR such a multiple-packets transmission scheme, we strive to ameliorate the performance of packet delay. The basic idea of this scheme is to enable P-nodes to transmit multiple packets towards different receivers in one transmission, and this transmission is implemented with hierarchical modulation to ensure sufficient SNR at all the receivers to demodulate their corresponding packets.

The rest of the paper is organized as follows. Starting with a brief introduction of DELAR in Section II, we elaborate the multiple-packets transmission scheme and the hierarchical modulation technique in Section III. We then evaluate the proposed scheme in Section IV. Finally, we conclude the paper in Section V.

\section{OVERVIEW OF DELAR}

We consider a mobile ad hoc network consisting of $N_{p}$ Pnodes and $N_{b}$ B-nodes, where $N_{p}$ and $N_{b}$ are system design parameters. We assume a single wireless broadcast channel shared by all the nodes, though DELAR can be easily extended to multi-channel cases. We also adopt a simple power control scheme as follows. Each B-node transmits omni-directionally and can maintain a circular transmission range $B T R$ (basic transmission range) before using up its battery, which can be properly set to achieve a good tradeoff between energy efficiency and network connectivity [3]. In addition, we postulate that P-nodes are able to adjust their transmission power so as to cover larger areas than Bnodes if needed. Moreover, all the P-nodes are assumed to have identical maximum transmission range of $P T R_{\max }=$ $M \times B T R$, where $M$ is a positive integer greater than 1. As revealed in [3], using common transmission power between the same type of nodes can ensure bidirectional links and thus the correct operations of existing routing and MAC protocols. With this simple yet efficient power control scheme, a unidirectional link only exists between a P-node and a B-node when they use different transmission power, instead of between any two B-nodes or P-nodes ${ }^{1}$. According to [4], such a simple power control scheme is believed to

\footnotetext{
${ }^{1}$ In this paper, we only consider asymmetric transmission power as the primary cause for unidirectional links and omit others such as various collision/noise/interference levels at different nodes.
}

be more practical than other expensive transmission power control schemes, either making unrealistic assumptions or having extra hardware requirements.

In order to better schedule the transmissions of $\mathrm{P}$ nodes and B-nodes, we adopt a time-division multiplexing method. We divide time into equal length time slots called Superframes. In each of the superframes, some time periods are exclusively designated to P-nodes, while the rest are shared by all P-nodes and B-nodes in the network. More specifically, during one cycle of the Superframe (see Fig. $1(\mathrm{a}))$, there is a $P$-to- $P$ period with length $t_{p p}$, in which only P-nodes are allowed to communicate with each other by using transmission range $T R_{p p}=m \times B T R(1<$ $m \leq M)$, while all B-nodes just keep silent, as if the network were merely formed by these "mobile core" Pnodes. Additionally, in one Superframe, each P-node has its own exclusive period called $P$-to- $B$ period with equal length $t_{p b}$, in which it can boost its transmission power to cover a range of $T R_{p b}=n \times B T R(1<n \leq M)^{2}$. Similar to the power control MAC (PCM) proposed in [5], P-nodes are assumed to be able to use $T R_{p b}$ for control frames and to adjust its transmission power to a certain lower level for data frames. The rest of one Superframe is called $B$-to- $B$ period with length $t_{b b}$ in which all the nodes in the network can contend for the channel and initiate transmissions towards nodes in their $T R_{b b}=B T R$. Obviously, all the P-nodes should act as common B-nodes in the $B-t o-B$ period by adjusting their transmission range back to $T R_{b b}$. Such a rendezvous of reservation-based and contention-based MAC schemes is able to schedule packet transmissions more efficiently.

Notice that during one $P$-to- $B$ period, since the P-node owning this period and the B-nodes it intends to communicate with have different transmission power, unidirectional links between them may be formed. Therefore, in contrast to the $P$-to- $P$ and $B$-to- $B$ periods where some common contention-based MAC protocols such as the IEEE 802.11 can be used, the $P$-to- $B$ period(s) demands an enhanced MAC protocol to support reliable communications over unidirectional links. Our Asymmetric MAC protocol A$M A C$ is exactly developed for this purpose. By introducing the mini-routing [1] into the MAC layer, A-MAC enables the receiver (e.g., node $C$ in Fig. 1(b)) to request the nodes on the backward path (e.g., $C \rightarrow B \rightarrow A$ in Fig. 1(b)) [1] to forward acknowledgement frames (e.g., CTS/ACK) back to the transmitter (e.g., node $A$ in Fig. 1(b)) at the MAC layer.

In DELAR, the heterogeneity of mobile nodes are also incorporated into the construction of routing tables. Routes

\footnotetext{
${ }^{2}$ To provide reliable communications during $P$-to- $B$ periods, usually $n$ is less than $m$.
} 


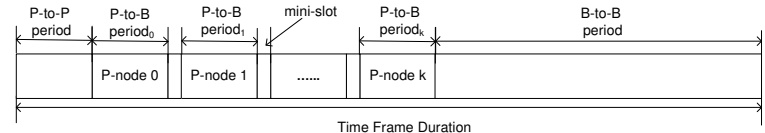

(a) The superframe structure.

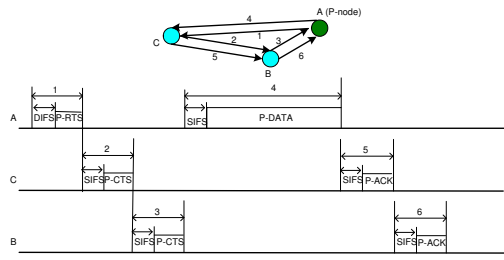

(b) The A-MAC operation procedure.

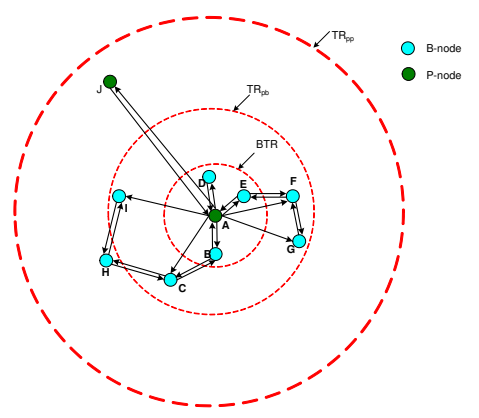

(c) An example.

Fig. 1. Illustration of DELAR.

are discovered based on routing metrics. For example, for node $i$, one of such metrics can be defined as Eq. 1, in which $\beta(i)=$ residual_energy $(i)-\mu \times$ queue_len $(i)$.

$$
\operatorname{cost}(i)= \begin{cases}1 / \beta(i), & \beta(i)>\gamma \\ a, & \beta(i) \leq \gamma\end{cases}
$$

We note that this metric takes into consideration of the residual energy and the load status of mobile nodes. Moreover, to reflect the device heterogeneity, different types of devices may have different values of $\gamma, \mu$ and $a$. Once generating a data packet, a node looks up its routing table and sends the data packet to the next hop as it does in common ad hoc routing protocols. If residing in the forwarding path and having received a forwarding request, a node will forward the data packet in an appropriate time period to the next hop according to its own routing table.

Fig. 1(c) shows an example when DELAR is used. For example, when P-node $J$ intends to send a packet to $\mathrm{B}$-node $C$, the rouing protocol will choose an energy efficient forwarding path: $J \rightarrow A \rightarrow C$. During one $P$-to$P$ period, P-node $C$ forwards the packet to another P-node $A$, further P-node $A$ will forward the packet to node $C$ in its $P$-to- $B$ period, and the reliable communication over the unidirectional link $A \rightarrow C$ is supported with the help from node $B$. For more details about DELAR, readers are referred to $[1]$.

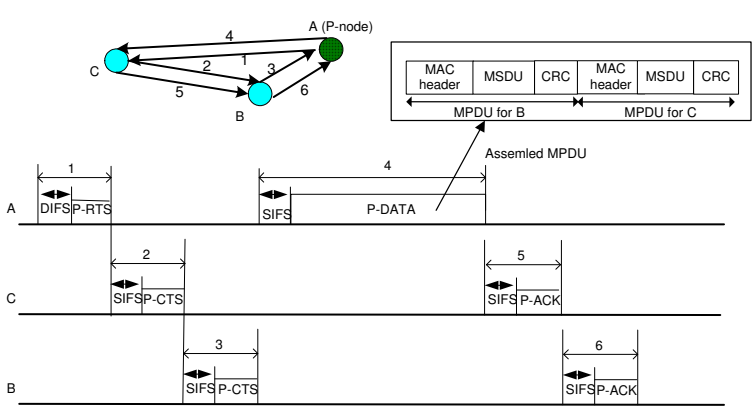

Fig. 2. The multiple-packets transmission enhancement to DELAR.

\section{Multiple-PaCkets Transmission}

In DELAR transmission scheduling is used to coordinate the transmission activities of P-nodes and B-nodes. One undesirable consequence is the excessive delay one packet may experience because it may be buffered at intermediate nodes to wait for appropriate transmission periods. On the other hand, since DELAR is energy aware and P-nodes are more likely to be chosen to forward a packet, lots of data packets may swarm to P-nodes. This may make P-nodes the "bottlenecks" of the network and further increase the delay that accumulated packets at P-nodes would experience. In what follows, we seek a way to at least alleviate this phenomenon.

\section{A. Multiple-packets Transmission}

In the basic design (see Fig. 1(b)), for example, $A$ can only transmit packets to either $B$ or $C$ each time. When P-node $A$ transmits a packet to $\mathrm{B}$-node $C$, node $B$ is only involved in forwarding the control frames (e.g., P-CTS/PACK). However, since the channel is reserved for P-node $A$ during its $P$-to- $B$ periods and node $B$ is also in P-node $A$ 's transmission range, node $B$ has the capability to receive and demodulate any signal transmitted by node $A$ if it is allowed. Therefor, if node $A$ can recognize B's capability and utilize it, i.e., transmitting packets to node $B$ and $C$ at the same time, the system performance could be improved. This multiple-packets transmission mechanism can be illustrated in Fig. 2. Suppose P-node $A$ has some packets to $B$ and some packets to $C$. Node $A$ would put one packet for $C$ and another for $B$ together, and send them in a single transmission from which nodes $B$ and $C$ can acquire their own part, respectively. In this way, we expect to see the improvement of the end-to-end delay performance of DELAR.

To do this, node $A$ first makes sure that both $B$ and $C$ are within its effective range $T R_{p b}$ after the P-RTS/P-CTS exchange as before. Then $A$ can pull out from the waiting queue one packet towards $C$ and another packet towards $B$, and send them in one P-DATA frame as depicted in Fig. 2. When seeing such a frame, nodes $B$ and $C$ can extract 


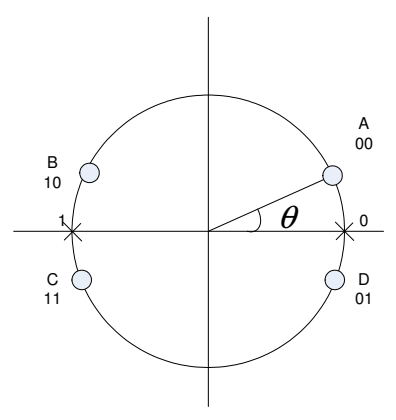

Fig. 3. General hierarchical 2/4-PSK constellation.

their own parts and dump the rest. The same procedures as specified in A-MAC are executed with the exception that node $B$ also needs to indicate in the P-ACK that it has successfully received the packet to itself. The similar procedure can be applied to the cases with more than one intermediate node on the backward paths.

With the above multiple-packets transmission mechanism, one may expect that the total number of packets that can be packed and transmitted at one time is bounded by the length of the Backward Path. Considering the possibility of adopting high-data rate modulation schemes with a higher power level so as not to degrade the received signal, more packets towards different receivers on the Backward Path can be assembled together and transmitted at the same time.

\section{B. Hierarchical Modulation}

To support the multiple-packets transmission, hierarchical modulation (or nonuniform modulation) schemes can be use to ensure all the receivers have enough received signal strength to demodulate the useful information. In hierarchical modulation schemes, the constellations consist of nonuniformly spaced symbols and allow for unequal error protection, i.e., different degrees of protection for transmitted bits within a symbol are allowed according to the importance of the information. For example, suppose that there are two streams of data, each of which has a priority (a target BER), and QPSK hierarchical modulation (see Fig. 3) is used. One bit from each stream is taken to form a symbol of two bits. The bit from bitstream with high priority (lower allowed BER) is assigned to the most significant position, e.g., the first bit in the constellation in Fig. 3. The bit from the bitstream with low priority is assigned to the less significant position. By adjusting the angle of $\theta$, the constellation can be determined to achieve the unequal error protection for each bitstream. Such unequal error protection has make hierarchical modulation very attractive in multimedia services [6]-[8].

We notice that in the previous research, the hierarchical modulation is used in the cases that the transmission power is fixed and the constellation is chosen in a way to allocate
Fig. 4. Implement multiple-packets transmission with hierarchical modulation

the power among multiple data streams to achieve unequal error protection. In addition, in the previous research, bitstreams usually are destined to the same receiver. However, in our DELAR scheme, the transmission power of P-nodes is not fixed. Moreover, when multiple-packets transmission is used, the bitstreams are destined to different receivers. As shown in Fig. 2, multiple receivers involved in the multiplepackets transmission have different distances to the transmitter. Therefore, in order to use hierarchical modulation to support multiple-packets transmission at a P-node, more power ought to be allocated to far away receivers and less power for close receivers. More specific, we need to find out the constellation and the minimum overall transmission power for a P-node so that all the receivers can successfully get their own packets. In the following we use QPSK hierarchical modulation to implement the multiple-packets transmission when two receivers are involved (Fig. 4).

Suppose $B E R_{\max }$ is the BER requirement for both receivers $B\left(d_{2}\right.$ away from transmitter $\left.A\right)$ and $C\left(d_{1}\right.$ away from transmitter $A$ ) to demodulate the received signal. Moreover, we assume two-ray ground model with $\alpha=4$ is used to model the radio propagation:

$$
P_{r}=\frac{P_{t} G_{t} G_{r} h_{t}^{2} h_{r}^{2}}{d^{4} L}
$$

where $\mathrm{L}$ is the system loss factor, $P_{t}$ is the transmission power, $P_{r}$ is the received power, $G_{t}\left(G_{r}\right)$ is the antenna gain at the transmitter(receiver), and $h_{t}\left(h_{r}\right)$ is the height of antenna at the transmitter(receiver). For simplicity, we assume all the nodes have the same antenna gain and height. We assume that $\theta$ is less than $45^{\circ}$ so that the inphase signal towards far away receiver $C$ is assigned with more power and quadrature signal towards close receiver $B$ with less power. It is easy to get the BER at $C$ as

$$
p_{1}=Q\left(\sqrt{2 \frac{P_{r 1} W}{N f}} \cos \theta\right)=Q\left(\sqrt{2 \frac{\frac{P_{t} G_{t} G_{r} h_{t}^{2} h_{r}^{2}}{d_{1}^{4} L}}{N f}} \cos \theta\right)
$$


where $N$ is the noise signal power, $f$ is the transmission bit rate, $W$ is the channel bandwidth (in $H z$ ), and $Q(\cdot)$ is Gaussian Q-function [7], [8]. Similarly, we can get the BER at the receiver $B$ as

$$
p_{2}=Q\left(\sqrt{2 \frac{P_{r 2} W}{N f}} \sin \theta\right)=Q\left(\sqrt{2 \frac{\frac{P_{t} G_{t} G_{r} h_{t}^{2} h_{r}^{2}}{d_{2}^{4} L}}{N f}} \sin \theta\right) .
$$

Thus, in order to correctly demodulate the received signal, the BER should satisfy the following conditions: $p_{i} \leq$ $B E R_{\max }$ where $i=1,2$. Since we are interested in the minimum transmission power of node $A$ when equality is satisfied: $p_{i}=B E R_{\max }$ where $i=1,2$, we define

$$
\gamma_{0}=Q^{-1}\left(B E R_{\max }\right)
$$

as the SNR achieving the $B E R_{\max }$ to facilitate us finding the boundary values of $P t$ and $\theta$. From Eq. 2 and Eq. 3, it is easy to get

$$
\sqrt{2 \frac{\frac{P_{t} G_{t} G_{r} h_{t}^{2} h_{r}^{2}}{d_{1}^{4} L} W}{N f}} \cos \theta=\gamma_{0}
$$

and

$$
\sqrt{2 \frac{\frac{P_{t} G_{t} G_{r} h_{t}^{2} h_{r}^{2}}{d_{2}^{4} L} W}{N f}} \sin \theta=\gamma_{0} .
$$

From Eq. 4 and Eq. 5, we can get

$$
\theta=\arctan \frac{d_{2}^{2}}{d_{1}^{2}}
$$

and

$$
P_{t \min }=\frac{\gamma_{0} N f\left(d_{1}^{4}+d_{2}^{4}\right) L}{W G_{t} G_{r} h_{t}^{2} h_{r}^{2}}
$$

Therefore, given the distances between the transmitter and receivers, we can get the minimum transmission power $P_{t \min }$ and $\theta$ in the QPSK hierarchical modulation constellation, and use this combination to implement the proposed multiple-packets transmission and achieve the BER requirements at both receivers. In practice, P-node $A$ may first use a predefined transmission power covering $T R_{p b}$, and transmit the RTS frame using typical BPSK. After the RTS-CTS handshake, $A$ can determine the two parameters $P_{t m i n}$ and $\theta$ based on the feedback from the two receivers. Then $A$ pulls out from the waiting queue one packet towards $C$ as the more important bitstream and one towards $B$ as the less important bitstream. Further it can transmit symbols using the aforementioned QPSK hierarchical modulation. We should note that, in order to avoid the possible interference when a $P$-to- $B$ period is shared by multiple P-nodes, the involved P-nodes, before enabling the multiple-packets transmission, should examine and make sure that $P_{\text {tmin }}$ is not greater than the power to cover $T R_{p b}$.

In fact, the minimum total transmission power to transmit the packets to each receiver separately using BPSK can be written as follows:

$$
P_{\text {ttoal }}=P_{t 1}+P_{t 2}=\frac{\gamma_{0} N f d_{1}^{4} L}{W G_{t} G_{r} h_{t}^{2} h_{r}^{2}}+\frac{\gamma_{0} N f d_{2}^{4} L}{W G_{t} G_{r} h_{t}^{2} h_{r}^{2}} .
$$

Compared Eq. 7 to Eq. 8, we can see that although $P_{t \min }$ alone is larger than either $P_{t 1}$ or $P_{t 2}, P_{t m i n}$ is identical to $P_{\text {ttoal }}$. This verifies that multiple-packets transmission with QPSK hierarchical modulation does not require extra transmission power ${ }^{3}$ compared to the case when multiplepackets transmission is not used. In fact, if multiple-packets transmission is implemented with uniform QPSK, in order to achieve the same BER requirement at both receivers, the transmission power would be $\sqrt{2} P_{t 1}$ which is much larger than $P_{\text {tmin }}$. Thus, in our case, QPSK hierarchical modulation is a rather reasonable option to implement multiple-packets transmission. Further, if we consider the energy used for control frames, the proposed multiplepackets transmission requires fewer control frames compared to the case when packets are transmitted separately. For example, in Fig. 2, transmission energy for three control frames (RTS/CTS/ACK) towards node $B$ can be saved when multiple-packets transmission is used.

\section{Performance Evaluation}

In order to evaluate the performance of DELAR, we implemented our scheme including the routing layer and the $A-M A C$ in the OPNET Modeler [9]. We simulated a network with 50 nodes randomly deployed in a $1500 \times 300 \mathrm{~m}^{2}$ area. The $B T R$ was $200 \mathrm{~m}$ and the channel bandwidth was $2 M$. In our simulations, all the nodes were capable of moving in the network according to the modified random Waypoint mobility model presented in [10]. The pause time was set to be zero in our simulations, meaning nodes were always moving. And each node moved with a randomly chosen speed between $\left[V_{\min }, V_{\max }\right]$, where $V_{\min }$ was fixed to be $1 \mathrm{~m} / \mathrm{s}$ and $V_{\max }$ assumed different values to reflect various network mobility levels.

There were 20 constant bit rate (CBR) data sessions between randomly selected source and destination pairs, and each source generated data packets of 512 bytes in length at a rate of 4 packets per second. In our simulation, B-nodes had the same initial energy reserve $5 k J$ and their transmission and reception power were $1560 \mathrm{~mW}$ and $930 \mathrm{~mW}$, respectively [11], while P-nodes had much larger initial energy reserve $30 k J$ and can adjust their transmission

\footnotetext{
${ }^{3}$ Here we only consider the power used to transmit the information bits.
} 
power as needed. The networks with $4 \mathrm{P}$-nodes were studied and these P-nodes were randomly deployed. Besides, we chose $m=4, n=2, t_{p p}=0.02 s, t_{p b}=0.02 s$, and $t_{b b}=0.05 \mathrm{~s}$. Six runs were carried out to get an average result for each simulation configuration and each run was executed for 900 seconds of simulation time.

Previous work [12], [13] has shown that the energy efficiency of routing protocols can be much improved by adopting such a routing metric as what we defined in Eq. 1. Therefore, we shall only compare our DELAR and DELAR with multiple-packets transmission (denoted by DELAR+MTP in Fig. 5) with the one referred to as EAR in this paper, which is developed for comparison purpose and in fact a variant of DSDV with the routing cost metric defined in Eq. 1. In EAR, all the P-nodes have the same transmission range as B-nodes. The following metrics will be adopted in comparison: average energy consumption defined as the total energy consumption of B-nodes for all packet transmissions and receptions normalized by the number of delivered packets; packet delivery ratio defined as the ratio of delivered data packets to those generated by the sources; average packet end-to-end delay defined as the average delay from when a packet is generated and transmitted by the source till it is received by the destination.

Fig. 5(a) compares the average energy consumption of DELAR with multiple-packets transmission, basic DELAR and EAR under different mobility levels. As we can see, DELAR always has less energy consumption than EAR because DELAR makes much better use of P-nodes than EAR through intelligent transmission scheduling and the allowance of P-nodes using different transmission power during various periods. DELAR with multiple-packets transmission can further reduce the energy consumption. It can be contributed to the reduced transmissions of control packets because multiple-packets transmission requires fewer control frames than separate transmissions. Generally the higher mobility leads to less energy consumption. After examining the average number of hops a packet may travel, we notice that higher mobility often results in shorter routes, which statistically leads to less energy consumption because fewer transmissions and receptions are involved.

As shown in Fig. 5(b), the packet delivery ratio decreases with the increase of the mobility, which is in accordance with previous studies. With the effective time-division scheduling DELAR always has higher packet delivery ratio than EAR in all kinds of mobility. The multiple-packets transmission can further improve the packet delivery ratio because transmitting multiple packets simultaneously can improve the channel utilization.

As the mobility increases, generally the delays increase.

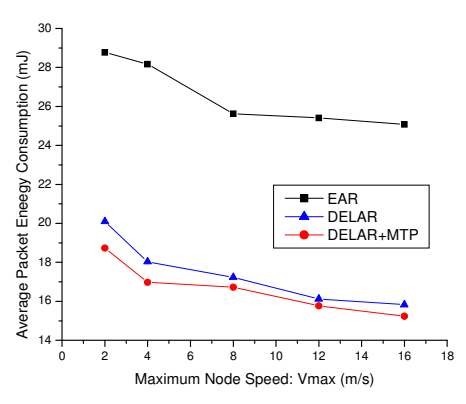

(a) Average energy consumption

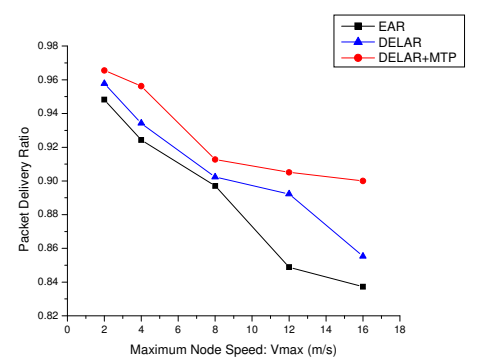

(b) Packet delivery ratio

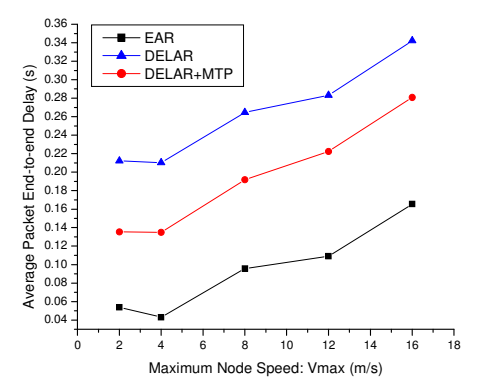

(c) Average packet end-to-end delay

Fig. 5. Simulation results with different maximum node speed.

However, DELAR has longer delay than EAR due to DELAR's time-division medium access control mechanism. But it is interesting to see that when multiple-packets transmission is used, the packet delay is much shorter than the delay when DELAR alone is used. This result validates that multiple-packets transmission can ameliorate the delay performance of DELAR. One interesting observation is that the delays fluctuate around $V_{\max }=2 \mathrm{~m} / \mathrm{s}$ and $V_{\max }=$ $4 \mathrm{~m} / \mathrm{s}$. This can be attributed to the used routing cost metric which causes many packets swarming to the P-nodes. This phenomenon results in longer waiting time at P-nodes. However, node movement helps alleviate such phenomenon by dispensing the traffic load.

\section{Conclusions And Future Work}

In this paper we proposed a multiple-packet transmission scheme to improve the performance of DELAR. In this scheme, powerful nodes are able to transmit multiple 
packets towards different receivers in one transmission, and this transmission is implemented with hierarchical modulation scheme to ensure BER requirements at all the receivers. Through simulation we validated that multiplepackets transmission not only improves the energy efficiency and packet delivery ratio beyond the basic DELAR, it also greatly shortens the packet delay caused by the timedivision scheduling. In our future work, we will study a more dynamic transmission scheduling scheme and take more factors into design consideration, e.g., QoS and security requirements.

\section{ACKNOWLEDGEMENTS}

This work was supported in part by the U.S. Office of Naval Research under Young Investigator Award N000140210464 and the US National Science Foundation under grant ANI-0093241 (CAREER Award).

\section{REFERENCES}

[1] W. Liu, Y. Zhang, W. Lou, and Y. Fang, "Delar: Device/energy/load aware relaying in heterogenous mobile ad hoc networks," in Proc. of MILCOM 2004, Montery, CA, October 2004.

[2] I. Chakeres and E. Belding-Royer, "Resource biased path selection in heterogeneous mobile networks," in UCSB Technical Report 2003-18, July 2003.

[3] V. Kawadia and P. R. Kumar, "Principles and protocols for power control in ad hoc networks," IEEE J. Select. Areas Commun., vol. 23, no. 5, pp. 76-88, January 2005, special Issue on Wireless Ad Hoc Networks.

[4] A. Muqattash and M. Krunz, "Powmac: A single-channel powercontrol protocol for throughput enhancement in wireless ad hoc networks," IEEE J. Select. Areas Commun., 2005, special Issue on Advances in Military Wireless Communications (to appear).

[5] E.-S. Jung and N. Vaidya, "A power control mac protocol for ad hoc networks," in Proc. of MobiCom'02, 2002.

[6] M.-S. Alouini, X. Tang, and A. Goldsmith, "An adaptive modulation scheme for simultaneous voice and data transmission over fading channels," IEEE J. Select. Areas Commun., vol. 17, no. 3, pp. 837-850, may 1999.

[7] M. Pursley and J. Shea, "Nonuniform phase-shift-key modulation for multimedia multicast transmission in mobile wireless networks," IEEE J. Select. Areas Commun., vol. 17, no. 9, pp. 774-784, May 1999.

[8] P. Vitthaladevuni and M.-S. Alouini, "Exact ber computation of generalized hierarchical psk constellations," IEEE Trans. on Wireless Comm., vol. 51, no. 12, pp. 2030-2037, December 2003.

[9] [Online]. Available: http://www.opnet.com

[10] J. Yoon, M. Liu, and B. Noble, "Sound mobility models," in Proc. of ACM MobiCom'03, San Diego, CA, September 2003.

[11] [Online]. Available: www.proxim.com/learn/library/datasheet/ 11abgcombocard.pdf

[12] S. Singh, M. Woo, and C. Raghavendra, "Power-aware routing in mobile ad hoc networks," in Proc. of MobiCom'98, 1998.

[13] C. Toh, "Maximum battery life routing to support ubiquitous mobile computing in wireless ad hoc networks," IEEE Commun. Mag., pp. 138-147, June 2001. 